\title{
On the Baldwin effect of He II emission lines in WR (WN) stars
}

\author{
J. I. van Gent ${ }^{1}$, H. J. G. L. M. Lamers ${ }^{1,2}$, A. de Koter $^{3}$, and P. W. Morris ${ }^{4}$ \\ 1 Astronomical Institute, Utrecht University, Princetonplein 5, 3584 CC Utrecht, The Netherlands \\ 2 SRON Laboratory for Space Research, Sorbonnelaan 2, 3584 CA Utrecht, The Netherlands \\ e-mail: lamers@astro.uu.nl \\ 3 Astronomical Institute "Anton Pannekoek", University of Amsterdam, Kruislaan 403, 1098 SJ Amsterdam, \\ The Netherlands \\ e-mail: dekoter@astro.uva.nl \\ 4 California Institute of Technology, SIRTF Science Center, M/C 100-22, Pasadena CA 91125, USA \\ e-mail: pmorris@ipac.caltech.edu
}

Received 24 July 2000 / Accepted 6 April 2001

\begin{abstract}
We investigate the relation between the equivalent width of He II emission lines and the monochromatic continuum luminosity at the line wavelength in the spectra of Wolf-Rayet stars. Model stellar atmospheres and spectra are used to show that the equivalent width inversely correlates with the monochromatic continuum luminosity. We find the effect in Wolf-Rayet star models over a large range of stellar parameters. The effect is shown to be connected to density differences among Wolf-Rayet star winds. The conclusion is drawn that the Baldwin effect may provide a new method for the determination of Wolf-Rayet star distances.
\end{abstract}

Key words. stars: atmospheres - stars: emission line - stars: Wolf-Rayet

\section{Introduction}

Spectral observations of Wolf-Rayet (WR) stars of the WN sequence in the optical, near infrared (NIR), and ultraviolet (UV), have shown an inverse proportionality between continuum luminosity and the line equivalent width of emission lines (Morris et al. 1993). This phenomenon was found among WN stars in the Large Magellanic Cloud, where distance and reddening properties are relatively well-determined. The relation between line and continuum luminosity is called the Baldwin effect after a similar correlation found in quasar spectra (Baldwin 1977). Morris et al. (1993) suggested the effect in WN stars to be the result of differences in wind density and effective radius among stars. If the Baldwin effect appears to be a general phenomenon in Wolf-Rayet spectra, it can be of great importance as it may indicate a way to derive absolute luminosities and distances from the spectra.

The determination of Galactic Wolf-Rayet star distances is known to be problematic, since a clear temperature-luminosity relation does not exist among stars with strong stellar winds (Conti \& Underhill 1988; van der Hucht 2000). The most reliable WR distances are

Send offprint requests to: J. van Gent, e-mail: gent@astro.uu.nl most often based on membership in OB associations and clusters and average reddening properties. Membership may be tenuous, though, and cluster or association distance can be uncertain. Less than one third of the known Galactic WR stars outside the Galactic Centre, however, appear in clusters or associations (van der Hucht 1988). Alternatively, distances can be estimated from a general trend of spectral subtype with absolute magnitude (based on members of clusters or associations or the Magellanic Clouds), but the spread in any one subtype bin is large, $\Delta M_{v} \simeq 2$ mag or more (e.g. Hamann \& Koesterke 1998) and suffers from low number statistics. Any independent method to derive distances should therefore be investigated. The Baldwin effect may provide such a method. In the Baldwin effect the continuum luminosity is affected by distance and reddening, but not the line equivalent widths. Moreover, the Baldwin effect of several emission lines can be used to determine the stellar distance from the equivalent width-luminosity relation.

In this paper we will investigate the underlying physics that causes the Baldwin effect in Wolf-Rayet stars. By means of model atmosphere calculations we will show the range of basic stellar parameters for which the Baldwin effect applies, and gain some insight into the intrinsic scatter 
in the equivalent width-luminosity relations. This knowledge is needed to assess the viability of the Baldwin effect as a distance estimation tool, and provides a new perspective on line and continuum coupling in terms of the fundamental stellar parameters. We will not try to give a ready to use formalism for the distance determination of WN stars, but restrict ourselves to explaining the physics behind the Baldwin effect. The absolute calibration of the Baldwin effect will be the subject of a future study, where we will introduce a scheme to apply the Baldwin effect as a spectroscopic method to estimate WN star distances in the Milky Way.

A simple explanation of the Baldwin effect from a theoretical viewpoint is given in Sect. 2; Sect. 3 describes the assumptions and the computer code used for the model atmospheres. The results of the calculations are presented in Sect. 4, which is followed by a general discussion and prospects for observational applications.

\section{A simple explanation of the Baldwin effect}

In this section we will use simple expressions in order to get an empirical understanding about the basic processes that determine the relation between the luminosity of the continuum and the equivalent width of spectral lines. We will discriminate between optically thick and thin winds. Also, we will assume that the atmosphere is in local thermodynamic equilibrium (LTE) and is isothermal at a temperature $T=T_{*}$. The reason why we first adopt LTE and a constant temperature is because we want to investigate the Baldwin effect in an analytical way. We will drop both assumptions in Sect. 4, where we present results of detailed non-LTE calculations.

\subsection{Optically thin wind}

We will first look at the case were the stellar wind is optically thin for both line and continuum radiation. In that case, the continuum radiation originates from the photosphere, roughly at the stellar radius $R_{*}$. We will call $R_{*}$ the core radius, describing the size of the star underlying the wind. If we consider an emission line transition with rest wavelength $\lambda_{0}$, the monochromatic continuum luminosity at $\lambda_{0}$ can be expressed as

$L_{\mathrm{c}}^{\lambda_{0}} \simeq 4 \pi R_{*}^{2} \pi B_{\lambda_{0}}\left(T\left(R_{*}\right)\right)$.

The energy emitted in the line and escaping from the atmosphere is given by the integral over the wind volume of the emissivity, corrected for the photons that are emitted towards the stellar core. The line luminosity is thus

$L_{\mathrm{l}}=4 \pi A_{\mathrm{ul}} h \nu_{0} \int_{R_{*}}^{\infty} n_{\mathrm{u}}(r) r^{2}\left[1-W_{\mathrm{D}}(r)\right] \mathrm{d} r$

where $n_{\mathrm{u}}$ is the population density of the upper level of the line transition, $\nu_{0}$ is the rest frequency of the transition, and $A_{\mathrm{ul}}$ is the corresponding Einstein coefficient for spontaneous emission. $W_{\mathrm{D}}$ is the geometrical dilution factor, which represents the angular size of the stellar core as seen from radial distance $r$ :

$$
W_{\mathrm{D}}=\frac{1}{2}\left\{1-\sqrt{1-\left(\frac{R_{*}}{r}\right)^{2}}\right\}
$$

In the following, we will ignore the factor $\left[1-W_{\mathrm{D}}(r)\right]$, for simplicity reasons. This will influence the luminosity by a factor of two, at most, and is not important for the qualitative description.

For recombination lines in an isothermal LTE atmosphere, the ratio $n_{\mathrm{u}} / \rho^{2}$ has a constant value over the wind volume. With the help of the equation of mass continuity,

$\dot{M}=4 \pi r^{2} \rho(r) v(r)$,

with mass-loss rate $\dot{M}$ and velocity field $v(r)$, we can express the line luminosity as

$$
\begin{aligned}
L_{\mathrm{l}} & =4 \pi A_{\mathrm{ul}} h \nu_{0} \frac{n_{\mathrm{u}}^{*}}{\left(\rho_{*}\right)^{2}} \int_{R_{*}}^{\infty} \rho(r)^{2} r^{2} \mathrm{~d} r \\
& =A_{\mathrm{ul}} h \nu_{0} \frac{n_{\mathrm{u}}^{*}}{\left(4 \pi \rho_{*}\right)^{2}}\left(\frac{\dot{M}}{v_{\infty}}\right)^{2} \frac{1}{R_{*}} E M_{1}
\end{aligned}
$$

with $\rho_{*}$ and $n_{\mathrm{u}}^{*}$ are the density and the upper level population at the core, respectively; $v_{\infty}$ the terminal wind velocity and we defined the dimensionless radius and velocity parameters: $x \equiv r / R_{*}$ and $w \equiv v / v_{\infty} . E M_{1}$ is the normalised emission measure for an optically thin wind, given by

$E M_{1}=4 \pi \int_{1}^{\infty} \frac{\mathrm{d} x}{x^{2} w^{2}}$.

For a given type of velocity law, the integral in Eq. (6) solves to a constant value, so we see that we can expect the line luminosity to scale as $L_{1} \propto \dot{M}^{2} v_{\infty}^{-2} R_{*}^{-1}$. The line equivalent width $W$ is the ratio $L_{\mathrm{l}} / L_{\mathrm{c}}$, so

$W \propto \dot{M}^{2} v_{\infty}^{-2} R_{*}^{-3} B_{\lambda_{0}}^{-1}\left(T\left(R_{*}\right)\right)$.

\subsection{Optically thick continuum and optically thin line}

For dense winds, as they occur in Wolf-Rayet stars, the continuum radiation field may originate at a radius $r_{\mathrm{c}} \equiv$ $R_{*} x_{\mathrm{c}}$ that is substantially larger than $R_{*}$. The exact value of this effective radius depends on the density and the observed wavelength. For now, we assume that the wind volume above $r_{\mathrm{c}}$ is still optically thin for line radiation, because of the high velocity gradient in the wind. The optically thick continuum and thin line limit is only expected to be valid for weak lines, i.e. not the diagnostic lines used to study the Baldwin effect. However, a discussion of this limit is important as it helps in understanding the more realistic case of optically thick continuum and partly optically thick line limit, presented in Sect. 2.3. 
The approximate expression for the continuum luminosity is given by:

$L_{\mathrm{c}}^{\lambda_{0}} \simeq 4 \pi R_{*}^{2} x_{\mathrm{c}}^{2} \pi B_{\lambda_{0}}\left(T\left(x_{\mathrm{c}}\right)\right)$.

For the line luminosity we get

$L_{\mathrm{l}}=A_{\mathrm{ul}} h \nu_{0} \frac{n_{\mathrm{u}}^{*}}{\left(4 \pi \rho_{*}\right)^{2}}\left(\frac{\dot{M}}{v_{\infty}}\right)^{2} \frac{1}{R_{*}} E M_{x_{\mathrm{c}}}$

in which the normalised emission measure is now determined by the wind volume above the dimensionless radius $x_{\mathrm{c}}$, where the continuum is formed:

$E M_{x_{\mathrm{c}}}=4 \pi \int_{x_{\mathrm{c}}}^{\infty} \frac{\mathrm{d} x}{x^{2} w^{2}}$.

The line equivalent width now scales as

$W \propto \dot{M}^{2} v_{\infty}^{-2} R_{*}^{-3} x_{\mathrm{c}}^{-2} B_{\lambda_{0}}^{-1}\left(T\left(x_{\mathrm{c}}\right)\right) E M_{x_{\mathrm{c}}}$.

The emission measure decreases as $x_{\mathrm{c}}$ increases. An increase of $x_{\mathrm{c}}$ occurs when the wind density is enhanced, either due to an increase of the mass-loss rate, a decrease of the stellar radius, or a decrease of the terminal velocity.

An estimate for the value of $x_{c}$ can be found if it is assumed that the continuum opacity is mainly free-free absorption. This assumption is reasonable for optical and infrared wavelengths (see e.g. Morris 1995, Chap. 4). In fact, at those wavelengths electron scattering may be the dominating factor in the continuum opacity, but as it neither creates nor destroys photons, it will not have a strong effect on the star's energy distribution (Lamers \& Waters 1984). Therefore we will ignore electron scattering in our determination of $x_{\mathrm{c}}$, in this simple description of the Baldwin effect. The opacities for free-free and boundfree processes can be expressed together as (Brussaard \& van de Hulst 1962)

$$
\begin{aligned}
\kappa_{\lambda}= & 1.370 \times 10^{-23} Z^{2}\left\{g_{\mathrm{ff}}(\lambda, T)+g_{\mathrm{bf}}(\lambda, T)\right\} \\
& \times \gamma n_{\mathrm{i}}^{2} \lambda^{3} T^{-\frac{1}{2}}\left(1-\mathrm{e}^{-\frac{h c}{\lambda \mathrm{k} T}}\right) \mathrm{cm}^{-1}
\end{aligned}
$$

with $Z$ the mean atomic charge, $n_{\mathrm{i}}$ the ion density, $\gamma$ the ratio of electron and ion density, and $g_{\mathrm{ff}}(\lambda, T)$ and $g_{\mathrm{bf}}(\lambda, T)$ the free-free and bound-free gaunt factors, respectively. For a spherically symmetric, extended atmosphere, the effective radius is the radius where the optical depth is about $\frac{1}{3}$, so from $\tau_{\lambda}\left(x_{\mathrm{c}}\right)=1 / 3$ we get

$C R_{*}^{-3}\left(\frac{\dot{M}}{v_{\infty}}\right)^{2} \int_{x_{\mathrm{c}}}^{\infty} T^{-\frac{1}{2}}\left(1-\mathrm{e}^{-\frac{h c}{\lambda \mathrm{k} T}}\right) \frac{\mathrm{d} x}{x^{4} w^{2}}=\frac{\lambda^{-3}}{3}$,

with $C$ a constant that depends on $Z, \gamma$, and the free-free and bound-free gaunt factors:

$C=8.676 \times 10^{-26} \frac{Z^{2}}{\mu^{2} m_{\mathrm{H}}^{2}} \gamma\left(g_{\mathrm{ff}}+g_{\mathrm{bf}}\right) R_{*}^{-3}$.

In this, $\mu$ is the mean ionic mass. In the derivation of Eq. (13), we assumed that the gaunt factors have a constant value over the wind volume, and also that $Z, \mu$,

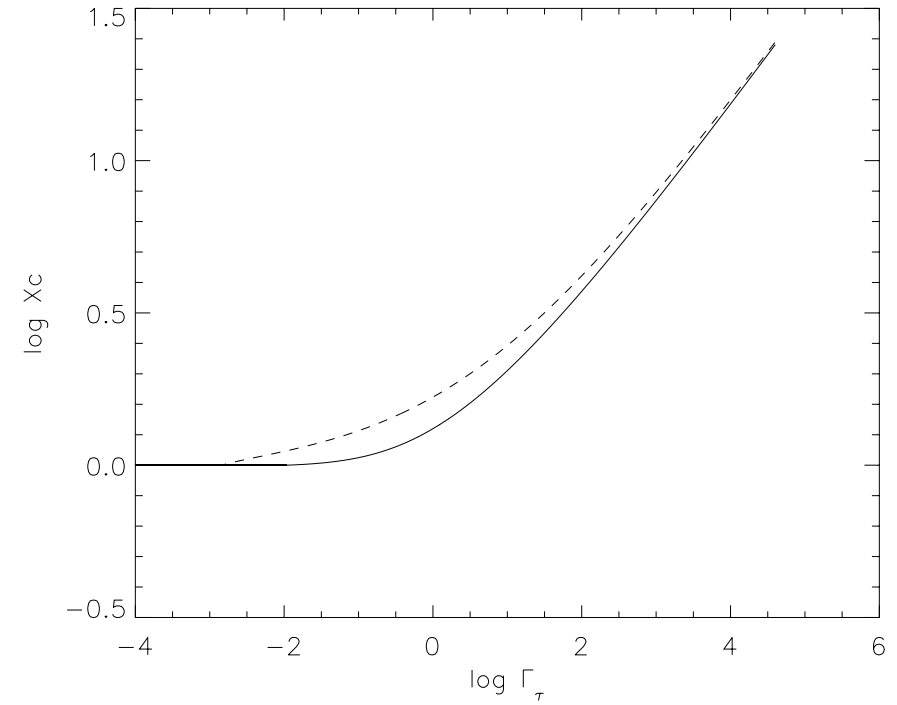

Fig. 1. Dimensionless effective radius, $x_{\mathrm{c}}=r_{\mathrm{c}} / R_{*}$, vs. optical depth parameter, $\Gamma_{\tau}$, for different $\beta$-type velocity laws. Full line: $\beta=1$, dotted line: $\beta=2 . \Gamma_{\tau}$ is defined by Eq. (16). For the figure, a wavelength value of $\lambda=1640 \AA$ is used.

and $\gamma$ are constants. Again, for simplicity, we will assume the atmosphere to be isothermal, with a temperature of $T=T_{*}$. Then Eq. (13) reduces to

$\int_{x_{\mathrm{c}}}^{\infty} \frac{\mathrm{d} x}{x^{4} w^{2}}=\frac{1}{\Gamma_{\tau}} \frac{\lambda^{-3}}{3}$,

where we defined

$\Gamma_{\tau}=C R_{*}^{-3}\left(\frac{\dot{M}}{v_{\infty}}\right)^{2} T_{*}^{-\frac{1}{2}}\left(1-\mathrm{e}^{-\frac{h c}{\lambda \mathrm{k} T_{*}}}\right)$.

Following Morris (1995), we will call the scaling factor $\Gamma_{\tau}$ the optical depth parameter.

We numerically calculated $x_{\mathrm{c}}$ from Eq. (13) with a $\beta$-type velocity law:

$v(r)=v_{\infty}\left(1-\frac{R_{*}}{r}\right)^{\beta}$.

For our qualitative description here, the value of $\beta$ is not important, and $\beta=1$ was adopted in Eqs. (8), (9), and (11) to determine line and continuum luminosities and equivalent widths.

Figure 1 shows the dependence of the normalised effective radius $x_{\mathrm{c}}$ on the optical depth parameter $\Gamma_{\tau}$, for different values of $\beta$. For the figure we used a wavelength of $\lambda=1640 \AA$ (the central wavelength of the He II 3-2 transition), typical gaunt factor values of $g_{\mathrm{ff}}=g_{\mathrm{bf}}=1$ (Waters \& Lamers 1984), and $\gamma=1$. We see that for thin winds (small $\Gamma_{\tau}$ ), the atmosphere becomes transparent, so $x_{\mathrm{c}}$ approaches unity. For dense winds, the continuum is formed at radii that are much larger than the stellar core. In those regions, the velocity does not differ much from the terminal velocity $(w \simeq 1)$. From Eq. (15) we then understand that for dense winds $\log x_{\mathrm{c}}$ increases proportional to $\log \Gamma_{\tau}$, with a slope of $\frac{1}{3}$. 

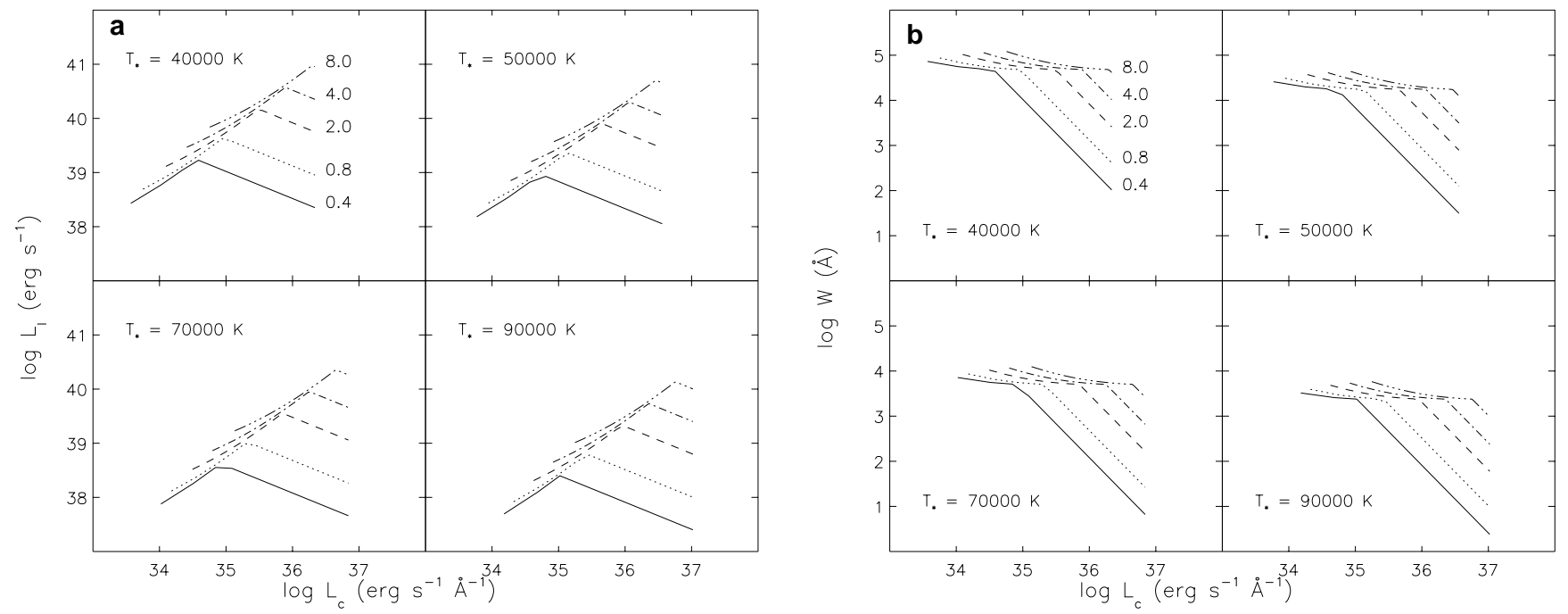

Fig. 2. Line luminosity $L_{1}($ panel a)) and equivalent width $W$ (panel b)) vs. continuum luminosity for the He II $\lambda 1640$ transition for isothermal atmospheres. Labels indicate values of $\dot{M} / v_{\infty}$, with the mass-loss rate in $10^{-5} M_{\odot} \mathrm{yr}^{-1}$ and the terminal velocity in $10^{3} \mathrm{~km} \mathrm{~s}^{-1}$.

In Fig. 2, the relationship is shown between line and continuum luminosity (panela) and between equivalent width and continuum luminosity (panelb), for different values of the stellar temperature $T_{*}$. Since we are interested in recombination lines, we will express the equivalent width of emission lines as positive values and ignore absorption dominated profiles throughout this paper.

The thick and thin wind regimes in the figure distinguish themselves by the sudden change in slope of the relations. For thin winds, where $x_{\mathrm{c}}=1$, the relation has a constant slope of $\alpha=-1 / 2$ in the $L_{\mathrm{l}}-L_{\mathrm{c}}$ plane and $\alpha=-\frac{3}{2}$ in the $W-L_{\mathrm{c}}$ plane. So for thin isothermal winds a Baldwin effect exists. For thick winds a Baldwin relation is still present, although the slope is rather flat. This is because of the influence of the effective radius on the wind volume that emits line radiation. However, an enhanced wind density due to an increase in mass loss rate at constant $R_{*}$ never increases $x_{\mathrm{c}}$ enough to lower the equivalent width. The slopes of the relations in Fig. 2 are not constant for the thick wind regime, due to the behaviour of $x_{\mathrm{c}}$ as function of the optical depth parameter, $\Gamma_{\tau}$.

\subsection{Optically thick wind for continuum and line}

In the more realistic case of a wind that is (partially) optically thick for both continuum and line radiation, the radiation observed around the central line wavelength originates further from the star than the continuum effective radius $r_{\mathrm{c}}$. Although the radius where the line radiation originates can depend strongly on the line of sight, we can at least find an estimate for the optical thickness of the atmosphere from the tangential Sobolev optical depth (e.g. Leitherer 1988; Lamers \& Cassinelli 1999, 203). In the Sobolev theory, it is assumed that a spectral line can emit photons of a certain observed frequency only over a very small region around the Sobolev point. This is due to Doppler shifting caused by the atmospheric outflow. Within this small volume the physical circumstances are not expected to change significantly, which simplifies the equations of radiative transfer. For a line of sight at impact parameter $p$, the tangential Sobolev optical depth is

$\tau_{\nu, \mathrm{p}}^{\mathrm{S}}=\frac{\pi e^{2}}{m_{\mathrm{e}} c} f_{\mathrm{lu}} \lambda_{0} n_{\mathrm{l}}\left(1-\frac{n_{\mathrm{u}}}{n_{\mathrm{l}}} \frac{g_{\mathrm{l}}}{g_{\mathrm{u}}}\right) \frac{r}{v}$

with all quantities evaluated at the Sobolev point. The variables $n_{1}$ and $n_{\mathrm{u}}$ are the number densities of the lower and upper level of the transition, respectively, $g_{\mathrm{l}}$ and $g_{\mathrm{u}}$ are the corresponding statistical weights, and $f_{\text {lu }}$ is the transition's oscillator strength.

Let us now assume that the continuum radiation originates again from the dimensionless radius $x_{\mathrm{c}}$, so the continuum luminosity is given by Eq. (8). If the atmosphere above $x_{\mathrm{c}}$ is optically thick for line radiation until a radius $x_{1}>x_{\mathrm{c}}$, the line emission is given by

$L_{\mathrm{l}}=L_{1}+L_{2}$

where $L_{1}$ is the line luminosity of the sphere of radius $x_{1}$ :

$L_{1}=4 \pi R_{*}^{2} x_{1}^{2} \pi S\left(x_{1}\right)$

with $S\left(x_{1}\right)$ the line source function at $x_{1} . L_{2}$ is the luminosity emitted in the optically thin layer above $x_{1}$ :

$L_{2}=A_{\mathrm{ul}} h \nu_{0} \frac{n_{\mathrm{u}}^{*}}{4 \pi \rho_{*}^{2}}\left(\frac{\dot{M}}{v_{\infty}}\right)^{2} \frac{1}{R_{*}} E M_{x_{1}}$.

If the total line luminosity is dominated by $L_{1}$, and we still assume an isothermal LTE atmosphere, then the equivalent width scales as $W \propto\left(x_{1} / x_{\mathrm{c}}\right)^{2}$, whereas if $L_{2}$ dominates, the equivalent width varies as

$W \propto \dot{M}^{2} v_{\infty}^{-2} R_{*}^{-3} x_{\mathrm{c}}^{-2} E M_{x_{1}}$ 
in which the expression for the emission measure is similar to Eq. (10), but with $x_{1}$ as the lower boundary for $x$. Since $x_{1}>x_{\mathrm{c}}$, we have $E M_{x_{1}}<E M_{x_{\mathrm{c}}}$, and therefore lower equivalent width values compared to the case of optically thin lines.

From the above description for different types of stellar atmospheres, we conclude that the Baldwin effect naturally follows from differences in wind density among stars, and will be generally present in both optically thin and optically thick atmospheres, albeit with different slopes. The main stellar parameters, core radius, mass-loss rate, terminal wind velocity, and stellar temperature, all play a role in the exact relation between line equivalent width and monochromatic continuum luminosity. The Baldwin relation of Wolf-Rayet stars is mainly caused by differences in core radius between stars. The relation is broadened by differences in temperature and the ratio of the mass-loss rate and terminal velocity. For a real sample of Wolf-Rayet stars, these three parameters are not fully independent, as the star's radiation field depends on radius and effective temperature and it is the radiation field that drives the mass loss rate. A given sample of Wolf-Rayet stars therefore will cover only part of the diagram shown in Fig. 2. However, our goal here is merely to show the influence of each of the basic stellar parameters on the relation between line equivalent width and continuum luminosity. A more realistic description follows from the detailed model atmosphere calculations, given below.

\section{Model atmospheres}

We calculated model stellar atmospheres to study the physical details behind the Baldwin effect and to be able to predict the relation between line equivalent width and continuum luminosity. For the synthetic atmospheres we used the non-LTE code ISA-Wind (de koter et al. 1993, 1997), that produces model atmospheres in a spherically symmetric geometry. Both the photospheric and the stellar wind structure are calculated (unified atmosphere), as opposed to the core-halo approach, in which the photospheric radiation field is defined as input at the base of the extended envelope of the model star.

The equations that describe the transfer of the continuum radiation through the atmosphere are solved under the constraint of statistical equilibrium, through the method of approximate lambda iteration. Here, we do not give a detailed description of this method, but instead refer to de koter et al. $(1993,1997)$. For the line radiation transfer an improved Sobolev approximation (ISA) is used, which takes into account the diffuse radiation field in the outflowing atmosphere, as well as continuum processes within the line resonance volume (Puls \& Hummer 1988).

\subsection{Chemical composition}

We calculated model atmospheres that consist purely of helium. WN stars are generally hydrogen depleted and, compared to helium, their nitrogen abundance is rather low, so the helium lines are not influenced much by the presence of other chemical elements (e.g. Hamann \& Koesterke 1998). This provides the purest way of studying the relation between continuum luminosity and line equivalent width for helium. The complexity of the He atom in the models is the same as used by de koter et al. (1997).

Besides thermal motions, for the calculation of the synthetic spectra no additional turbulent velocity fields that could broaden the line profiles were taken into account in the calculation of the synthetic spectra. This way, the line equivalent width of both optically thin and thick lines is completely due to the bulk wind velocity field and thermal motions. We will discuss the implications of the simplifications made here in Sect. 5 .

\subsection{Atmosphere structure}

The photospheric density structure in each model is determined by an inner radius, $R_{*}$, which determines the size of the core underlying the atmosphere, the effective gravity acceleration, $g_{\text {eff }}$, and the density at the core, $\rho_{*}$. The latter is chosen such, that the total Rosseland optical depth, $\tau_{\mathrm{R}}$, has a value between 20 and 25 . Following Hamann (1985), and subsequent papers, we set the scale height in the photosphere by assuming $\log \left(g_{\text {eff }}\right)=3.5$. With the above parameters setting the boundary conditions, the density structure then follows from the solution of the momentum equation for a stationary wind. This equation is given by

$v \frac{\mathrm{d} v}{\mathrm{~d} r}+\frac{1}{\rho} \frac{\mathrm{d} p}{\mathrm{~d} r}+\frac{G M(1-\Gamma)}{r^{2}}=0$

with velocity field $v$, gas pressure $p$, and stellar mass $M$. Radiative acceleration by electron scattering is included in the equation by the parameter $\Gamma$, which denotes the ratio between radiative and gravitational acceleration:

$\Gamma=\frac{g_{\mathrm{rad}}}{g_{\mathrm{N}}}=\frac{\sigma_{\mathrm{e}} L}{4 \pi c G M}=7.66 \times 10^{-5} \sigma_{\mathrm{e}}\left(\frac{M}{M_{\odot}}\right)^{-1}\left(\frac{L}{L_{\odot}}\right)$

where $L$ is the stellar luminosity and $\sigma_{\mathrm{e}}$ is the Thomson scattering coefficient. In the subsonic region the velocity structure follows from the density structure by using the equation of mass continuity (Eq. (4)).

In the wind region a $\beta$-type velocity law is used

$v(r)=v_{\infty}\left(1-\frac{r_{0}}{r}\right)^{\beta}$

and the corresponding density structure follows from mass continuity. The photosphere and wind regions are smoothly connected by requiring continuity of both the velocity field and its gradient. These conditions are met by iteratively solving for the radius where the two regions 
Table 1. He II transitions for which the Baldwin effect was studied.

\begin{tabular}{ll}
\hline Central wavelength $(\AA)$ & Levels $n-m$ \\
\hline 1640 & $3-2$ \\
3203 & $5-3$ \\
4686 & $4-3$ \\
5411 & $7-4$ \\
10124 & $5-4$ \\
\hline
\end{tabular}

are connected, $r_{\text {con }}$ and the parameter $r_{0}$ in Eq. (25). It turns out that $r_{\text {con }}$ lies close to the sonic radius.

The luminosity is specified by the stellar temperature, $T_{*}$, as

$L=4 \pi R_{*}^{2} \sigma T_{*}^{4}$,

where $T_{*}$ corresponds to the effective temperature that describes the flux at the core radius, $R_{*}$, of the model. $T_{*}$ is not the real effective temperature $T_{\text {eff }}$, as $R_{*}$ is not the effective stellar radius. A unique radius is hard to define, as it is intrinsically wavelength dependent. We will define the effective radius, $R_{\text {eff }}$, as the radial distance where the thermalization optical depth equals $1 / \sqrt{3}$ at $\lambda=5500 \AA$ (Schmutz et al. 1990). This radius thus follows from the model output, as does $T_{\text {eff }}$, through the luminosity and the radius.

The temperature structure follows from the assumption of radiative equilibrium in a grey atmosphere in LTE. We allow the temperature to drop with radius down to a minimum temperature $T_{\min }$, after that it remains constant. For $T_{\min }$ we use the value of $T_{*} / 2$, suggested for O-stars by Drew (1989). Note however that there are indications that for Wolf-Rayet stars lower values for $T_{\min }$ are more realistic (e.g. Hamann et al. 1994).

\subsection{Convergence}

Most models were found to converge well. As a criterium for convergence we required that for two succeeding iteration steps the populations of all atomic levels at all radii not to differ by more than $0.1 \%$.

For some models this criterium was not met; those models kept "flipping" between two solutions that generally differed less than 1 percent. In those cases it was also found that the two solutions result in almost identical synthetic spectra, with less than one percent difference in flux levels at all wavelengths. Therefore we included those cases in our model sample.

\subsection{Synthetic spectra}

We calculated synthetic spectra by solving the radiative transfer equations through the model atmospheres. We selected five He II transitions as being suitable for studying the Baldwin effect. These lines are listed in Table 1.
Table 2. Input parameters of the ISA-Wind model atmospheres.

\begin{tabular}{l|l}
\hline$R_{*}\left(R_{\odot}\right)$ & $1,2,3,5,10,15,20,25$, \\
& $27,28,29,30$ \\
$T_{*}(\mathrm{kK})$ & $10,20,30,40,50,60$, \\
& $70,80,90$ \\
$v_{\infty}\left(\mathrm{km} \mathrm{s}^{-1}\right)$ & $1250,2000,2500$ \\
$\dot{M} / v_{\infty}\left(10^{-5} M_{\odot} \mathrm{yr}^{-1}\right) /\left(10^{3} \mathrm{~km} \mathrm{~s}^{-1}\right)$ & $0.4,0.8,2,4,8$ \\
$\log g$ & 3.5 \\
$\beta$ & 1 \\
\hline
\end{tabular}

The reason for selecting these lines is that they belong to the strongest He II lines in observed Wolf-Rayet star spectra, and that they are relatively free of blends by other lines. For example, in many observed Wolf-Rayet spectra the He II Balmer lines from even numbered upper levels suffer from blends with the hydrogen Lyman series. This may hamper the applicability of the Baldwin effect in those WN stars that have still significant hydrogen left (i.e. mostly the WNL-type stars).

In both the calculations of the atmosphere and the spectrum, the effects of electron scattering in lines are not included. The inclusion of electron scattering processes can significantly increase the equivalent width of optically thick lines as the scattering of line photons reduces the line optical depth. However, in our calculations we ignore the effect as we are currently only interested in the qualitative explanation of the Baldwin effect, which is unlikely to be altered by electron scattering processes.

Other simplifications in our modelling that should be mentioned are the assumptions of (i) spherical symmetry and (ii) a homogeneous density distribution (i.e. we ignore the effects of clumping). We will discuss these assumptions in more detail in Sect. 5.

\section{The synthetic Baldwin effect}

\subsection{Grid parameters}

We calculated a large grid of atmosphere models. The stellar temperature, $T_{*}$, ranges from 10 to $90 \mathrm{kK}$, and the core radius $R_{*}$ from 1 to $30 R_{\odot}$. This way both parameters cover the range that is thought to occur in real WN stars (Hamann \& Koesterke 1998). The parameters of our model grid are presented in Table 2.

\subsection{Spectra}

Most lines in our model spectra show pure emission profiles, resulting from recombination processes followed by cascading events. For the cooler models, the lines may contain a P Cygni component, due to scattering processes. The equivalent width of each line was measured over the full profile. As long as the scattering region of the atmosphere around the star is large compared to the stellar 
a

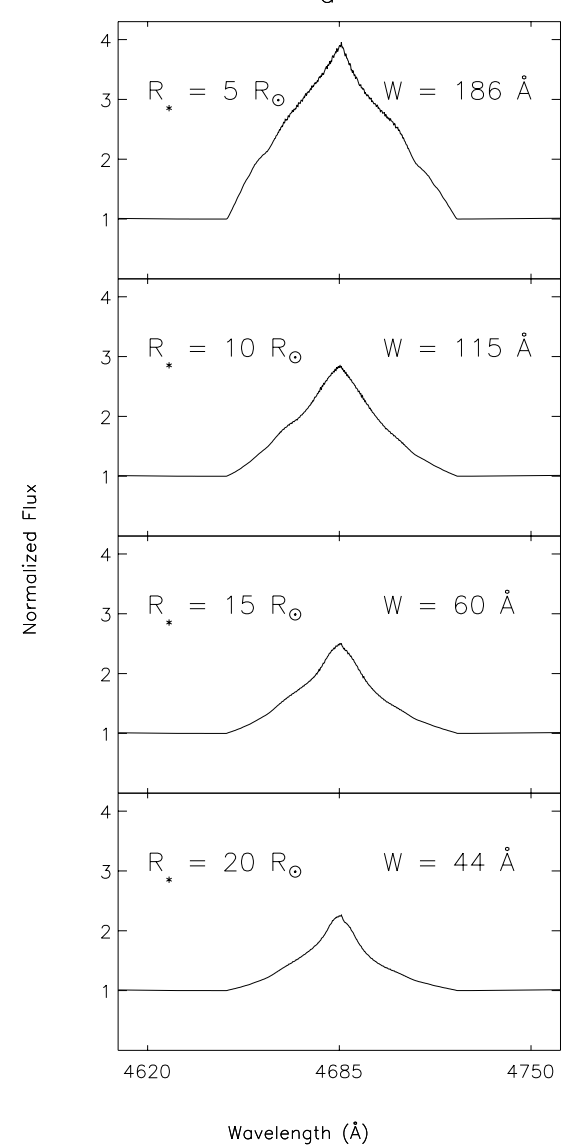

b

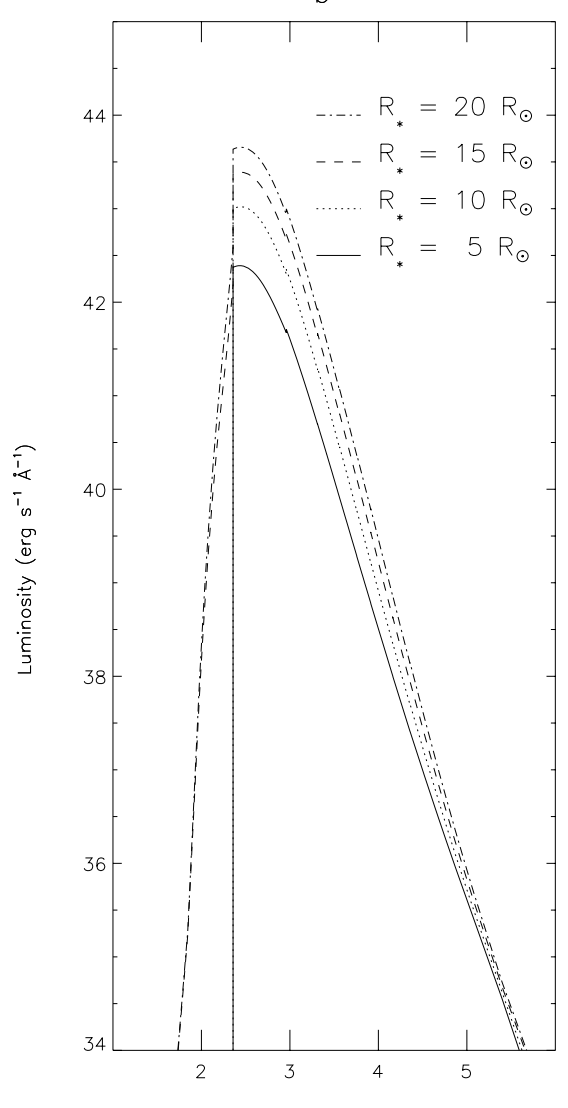

Log Wavelength $(\stackrel{\AA}{)}$

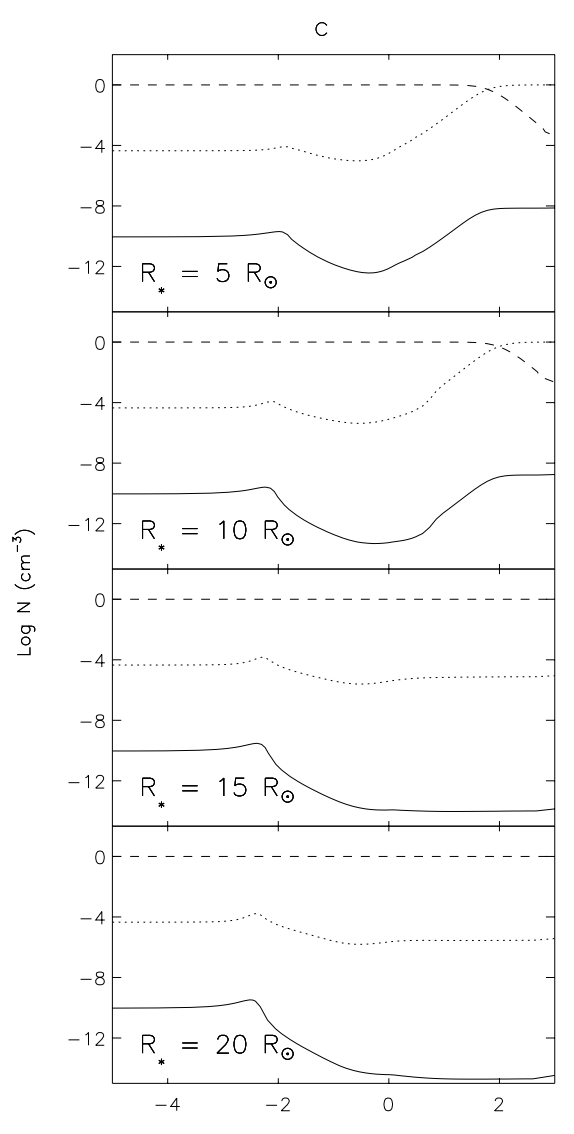

Log height $(R$ )

Fig. 3. Four models that show the Baldwin effect. Panel a) Continuum normalized line spectra of He II $\lambda 4686$ for different core radii. $W$ indicates the line equivalent width in $\AA$. Panel b) The corresponding spectral energy distributions. Panel c) Ionization structure of the same models, as function of height above the core. Full line: He I; dotted line: He II; dashed line: He III. In all three panels, $R_{*}$ indicates the core radius. For all models: $T_{*}=60 \mathrm{kK}, \dot{M}=5 \times 10^{-5} M_{\odot} \mathrm{yr}^{-1}$, and $v_{\infty}=2500 \mathrm{~km} \mathrm{~s}^{-1}$. For increasing core radius, the continuum luminosity increases, whereas the line equivalent width decreases: the Baldwin effect.

core size, only few photons will be scattered back to the stellar core and lost from the radiation field. The absorption component will thus be of about equal size as the scattering emission component. The net equivalent width, measured over the full line profile, therefore is a good representation of the equivalent width of the emission profile formed by recombination processes.

As can be seen from Table 2, we used three different values of the terminal velocity $v_{\infty}$ in our model atmospheres, such that for each value of $v_{\infty}$ the same set of values of $\dot{M} / v_{\infty}$ was obtained. A well known phenomena in synthetic spectra of Wolf-Rayet stars is that models with the same so called transformed radius show approximately the same normalized spectrum. This was found by Schmutz et al. (1989). The transformed radius is given by

$R_{\mathrm{t}}=R_{*} \times\left(\frac{v_{\infty}}{2500 \mathrm{~km} \mathrm{~s}^{-1}} / \frac{\dot{M}}{10^{-4} M_{\odot} \mathrm{yr}^{-1}}\right)^{\frac{2}{3}}$

One therefore expects a similar Baldwin effect for star samples that have the same value of the ratio $\dot{M} / v_{\infty}$.

\subsection{Ionization}

A major role in the presence of the Baldwin effect and its characteristics is played by the ionization structure in the atmosphere. In our simple explanation of the Baldwin effect (Sect. 2), we assumed an isothermal atmosphere in LTE, so the stratification of the ionization was only determined by the density. In a real Wolf-Rayet star atmosphere the temperature structure drops with radial distance, so a more complex ionization stratification is to be expected. Based on the dependence of the $W-L_{\text {c }}$ relations on the basic stellar parameters, a sample of stars should have a similar degree of ionization over the line forming region in their atmospheres, in order to show a Baldwin effect. Since the degree of ionization depends on mass density, temperature, and the radiation field, an arbitrary sample of Wolf-Rayet stars will expose a range of ionization structures. To illustrate the influence of the ionization structure, Figs. 3 and 4 show some line profiles along with the spectral energy distribution (SED) and helium ionization structure of the corresponding model atmospheres. Figure 3 shows models with stellar temperature $T_{*}=60 \mathrm{kK}$. From top to bottom the core radius increases from $5 R_{\odot}$ to $20 R_{\odot}$. 
a

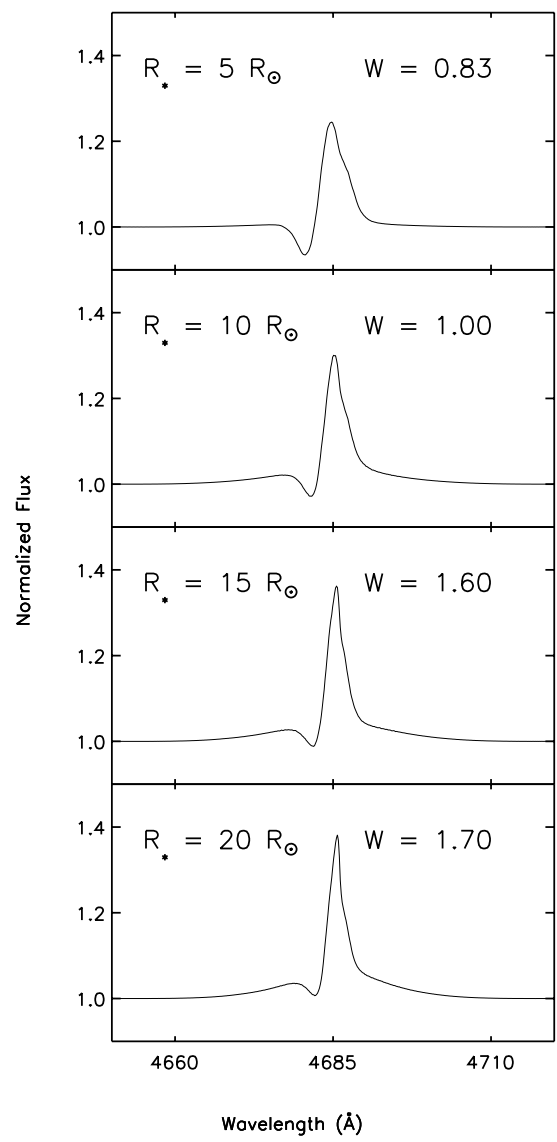

b

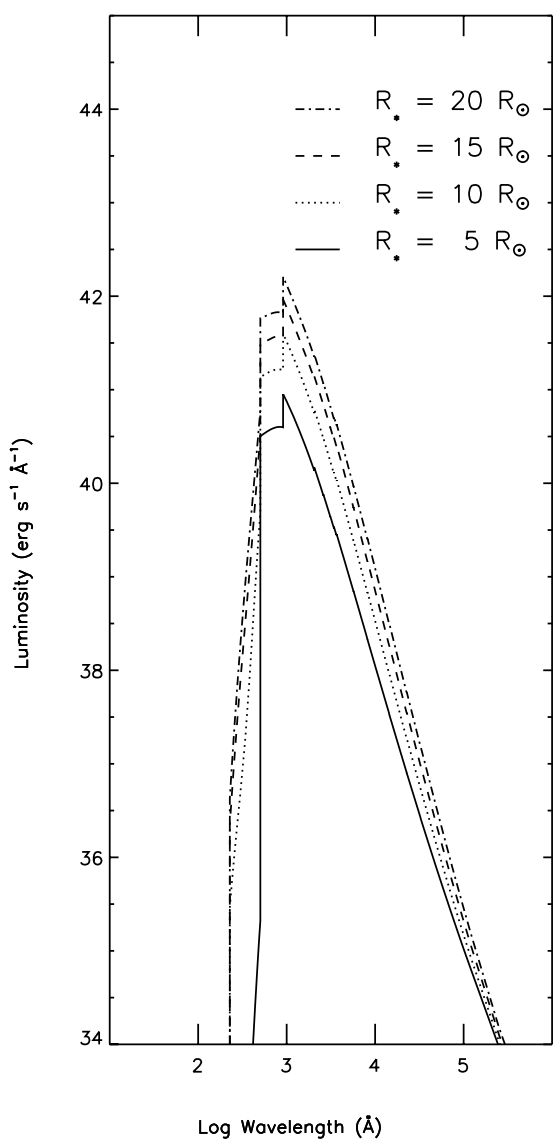

C

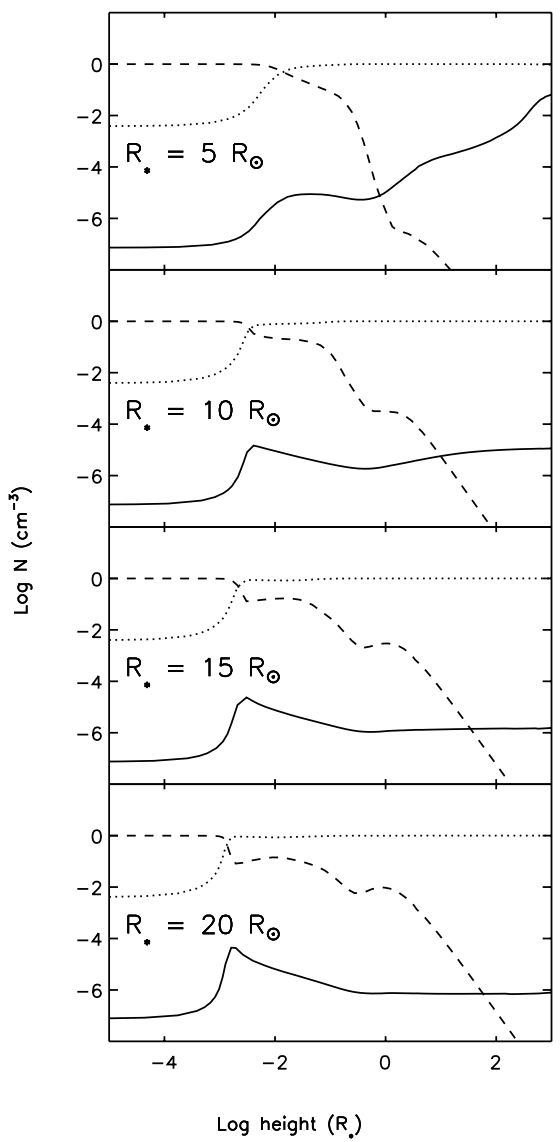

Fig. 4. Four models that do not show the Baldwin effect. For all models: $T_{*}=30 \mathrm{kK}$ and $\dot{M}=1 \times 10^{-5} M_{\odot} \mathrm{yr}^{-1}$. For explanation of the labels: see text with Fig. 3 .

From the line profiles and the values of equivalent width and monochromatic continuum luminosity we see that this small sample of models shows a Baldwin effect. Indeed, as already seen in Sect. 2, the equivalent width is inversely proportional to the monochromatic continuum luminosity, whereas the integrated line luminosity scales directly proportional to it. From the ionization stratifications we see that helium is fully ionized over a large volume of the wind. We can therefore understand the occurrence of a Baldwin effect from the explanation in Sect. 2. Recombination of He III to He II only takes place in the outer regions of the wind, where it no longer influences the line profiles.

At a stellar temperature of $T_{*}=30 \mathrm{kK}$ (Fig. 4) the relation between line equivalent width and continuum luminosity is opposite from a Baldwin effect. At this low temperature He III is not the dominant ionization stage in the line forming wind regime. Emission due to recombination therefore is much less than at $T_{*}=60 \mathrm{kK}$. Furthermore, towards larger core radii, the ionization balance shifts in favour of He III, as a result of reducing wind density. Therefore at larger core radii there is more line emission, leading to an increase in equivalent width with core radius, as opposed to a decrease at higher values of $T_{*}$.

\subsection{Equivalent widths and luminosities}

In Sect. 2 we used simple expressions to determine the relation between line luminosity and equivalent width on one hand and the monochromatic continuum luminosity on the other hand (Fig. 2). Figures 5 to 9 show similar relations for line equivalent width versus continuum luminosity, but this time from the model atmosphere calculations and for the five helium lines He II $\lambda 1640$, He II $\lambda 3203$, He II $\lambda$ 4686, He II $\lambda$ 5411, and He II $\lambda 10124$. Each panel shows models for a certain stellar temperature, $T_{*}$, but for different values of the mass-loss rate, $\dot{M}$, and core radius, $R_{*}$. For clarity, we only show the models with $v_{\infty}=2500 \mathrm{~km} \mathrm{~s}^{-1}$. For other values of $v_{\infty}$ the relations are almost the same, because the spectrum is invariant for $R_{*}\left(\dot{M} / v_{\infty}\right)^{2 / 3}$.

From the figures we see that there is a Baldwin effect present in our model stars, although not over the whole parameter range. For the regions in parameter space where a Baldwin effect occurs the results agree very well with our simple estimates in Sect. 2, in a qualitative way. The model atmospheres confirm that the Baldwin effect is mainly caused by differences in wind density among the stars. However, there are two important differences between the result of these simple predictions and those from the detailed model atmospheres. Both are caused 


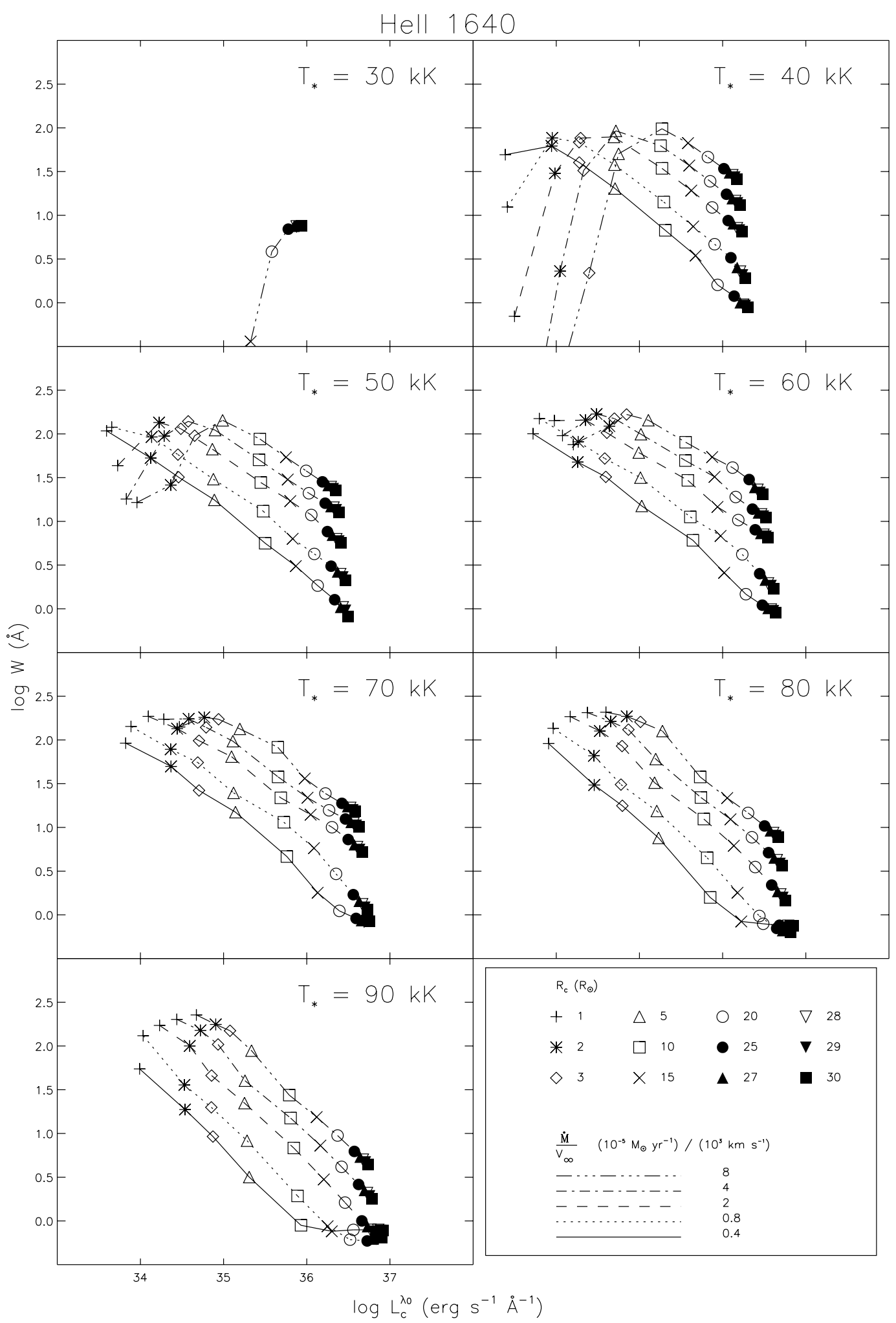

Fig. 5. Line equivalent width vs. monochromatic continuum luminosity for the He II $\lambda 1640$ line. Each panel shows $\log (W)$ as function of $\log \left(L_{\mathrm{c}}\right)$ for models with the same value of $T_{*}$, and for different values of the mass-loss rate, $\dot{M}$, and the core radius, $R_{*} . v_{\infty}=2500 \mathrm{~km} \mathrm{~s}^{-1}$. Models with the same value of $\dot{M}$ are connected by lines; the values of $R_{*}$ are indicated by symbols. Line style and symbols are explained in the bottom right panel.

by the fact that the model atmospheres are not isothermal, but have a more realistic temperature stratification that drops with radial distance. First of all, the Baldwin effect breaks down for models with low stellar temperature and high wind density. The reason for this is the ionization structure, which plays a major role in the presence of 


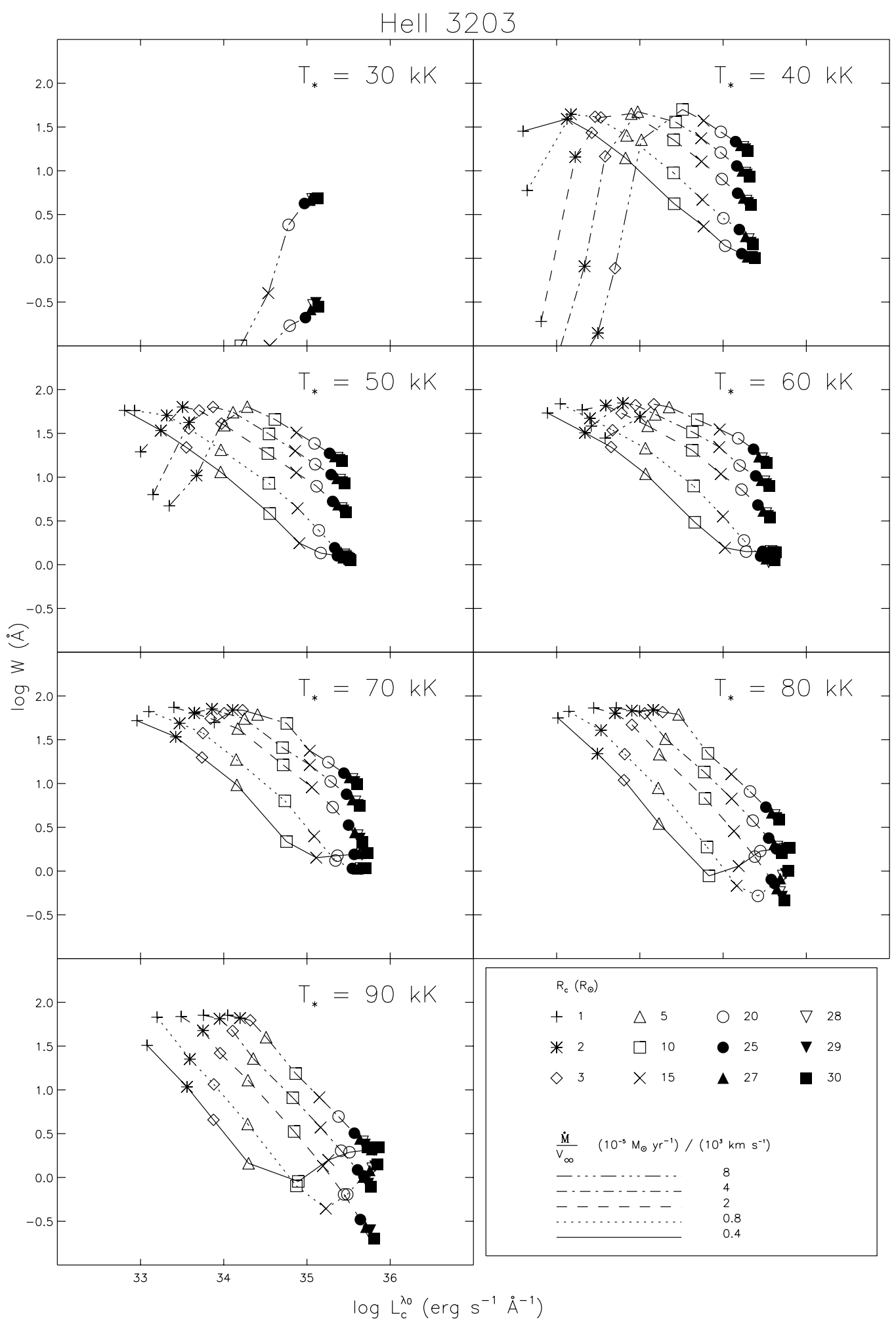

Fig. 6. Line equivalent width vs. monochromatic continuum luminosity for the He II $\lambda 3203$ line. For explanation, see the text below Fig. 5 .

the Baldwin effect. As mentioned above, at relatively low temperatures and high densities the dominant ionization stage shifts from He III to He II, reducing the emission of the He II lines.
The second important difference with the simple results of Sect. 2 is that there is no Baldwin effect for the models with the least dense winds in our grid. Again this is caused by the fact that the model atmospheres are non 


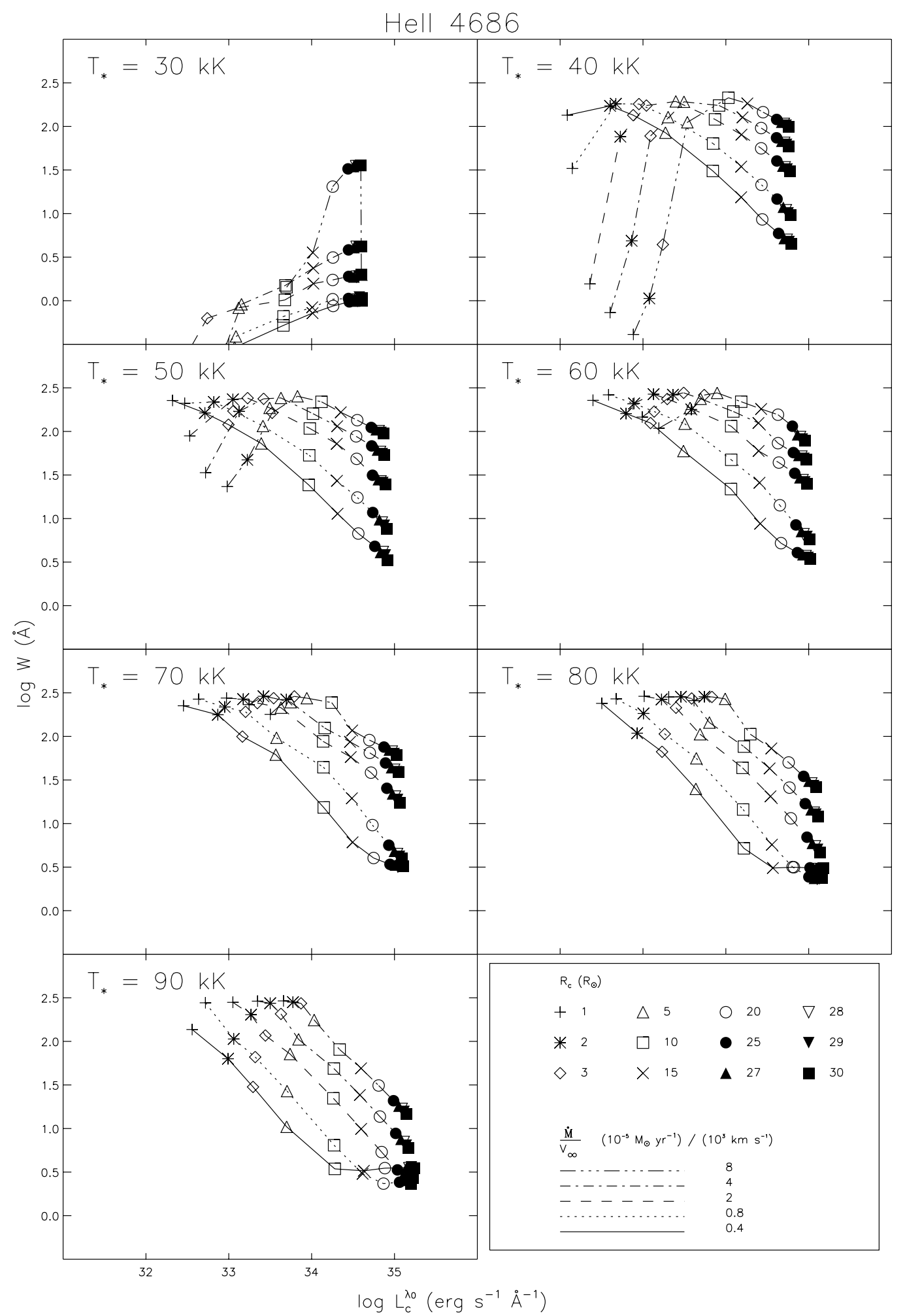

Fig. 7. Line equivalent width vs. monochromatic continuum luminosity for the He II $\lambda 4686$ line. For explanation, see the text below Fig. 5 .

isothermal. At the lowest mass-loss rates, in going from small to large core radius, the stellar wind can become optically thin for continuum radiation (cf. Fig. 1). The steep increase in temperature close to the photosphere then causes an enhanced line emission as compared to the optically thick winds. This disrupts the Baldwin-relation.

Besides these differences, the detailed calculations shown in Fig. 5 also confirm some important results from 


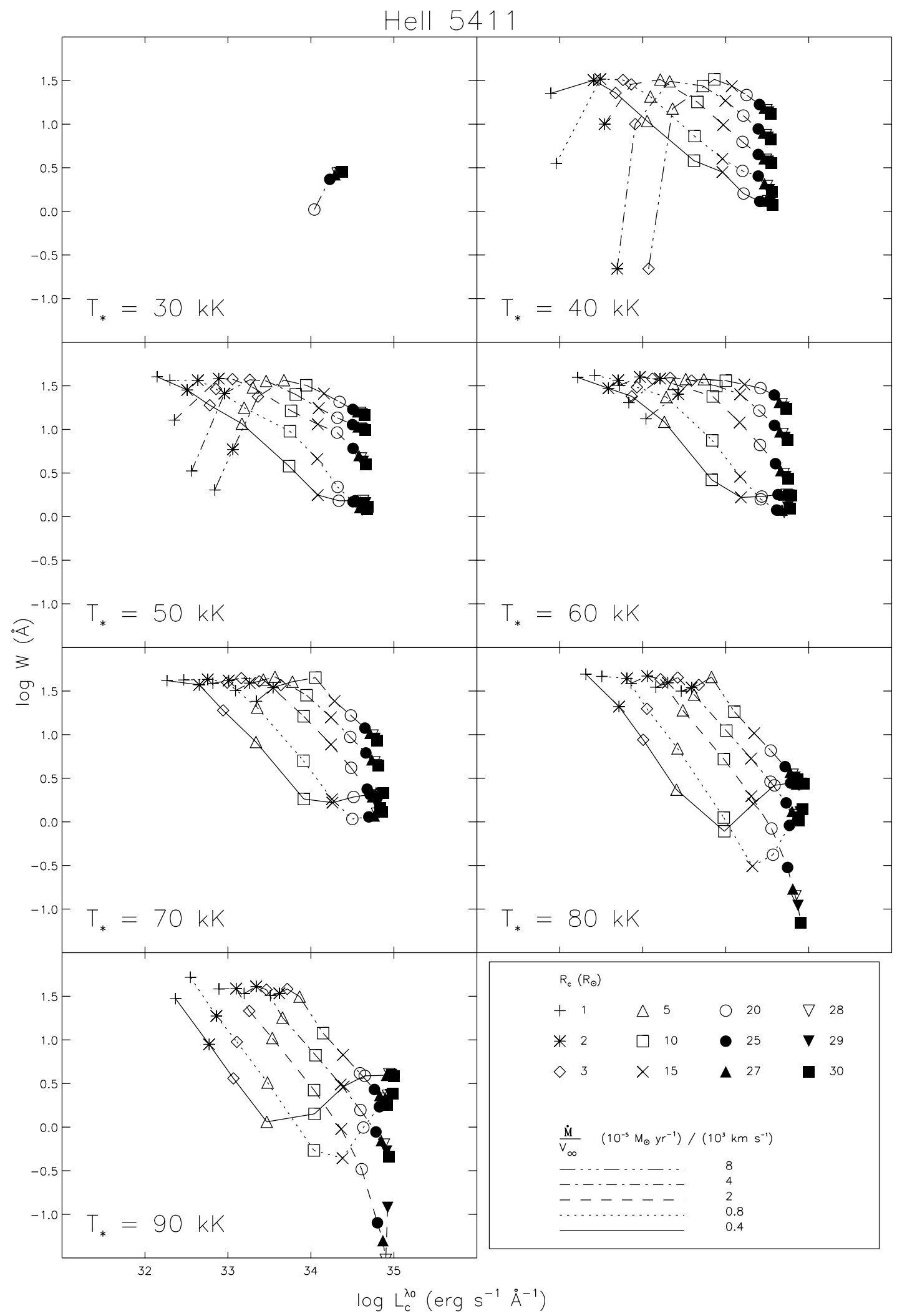

Fig. 8. Line equivalent width vs. monochromatic continuum luminosity for the He II $\lambda 5411$ line. For explanation, see the text below Fig. 5 .

the simple picture drawn in Sect. 2. In the parameter range where the Baldwin effect applies, differences in wind density among models due to different core radii cause the
Baldwin effect; a density enhancement (either due to increasing mass-loss rate or decreasing core radius) increases the line equivalent width even though this causes the 


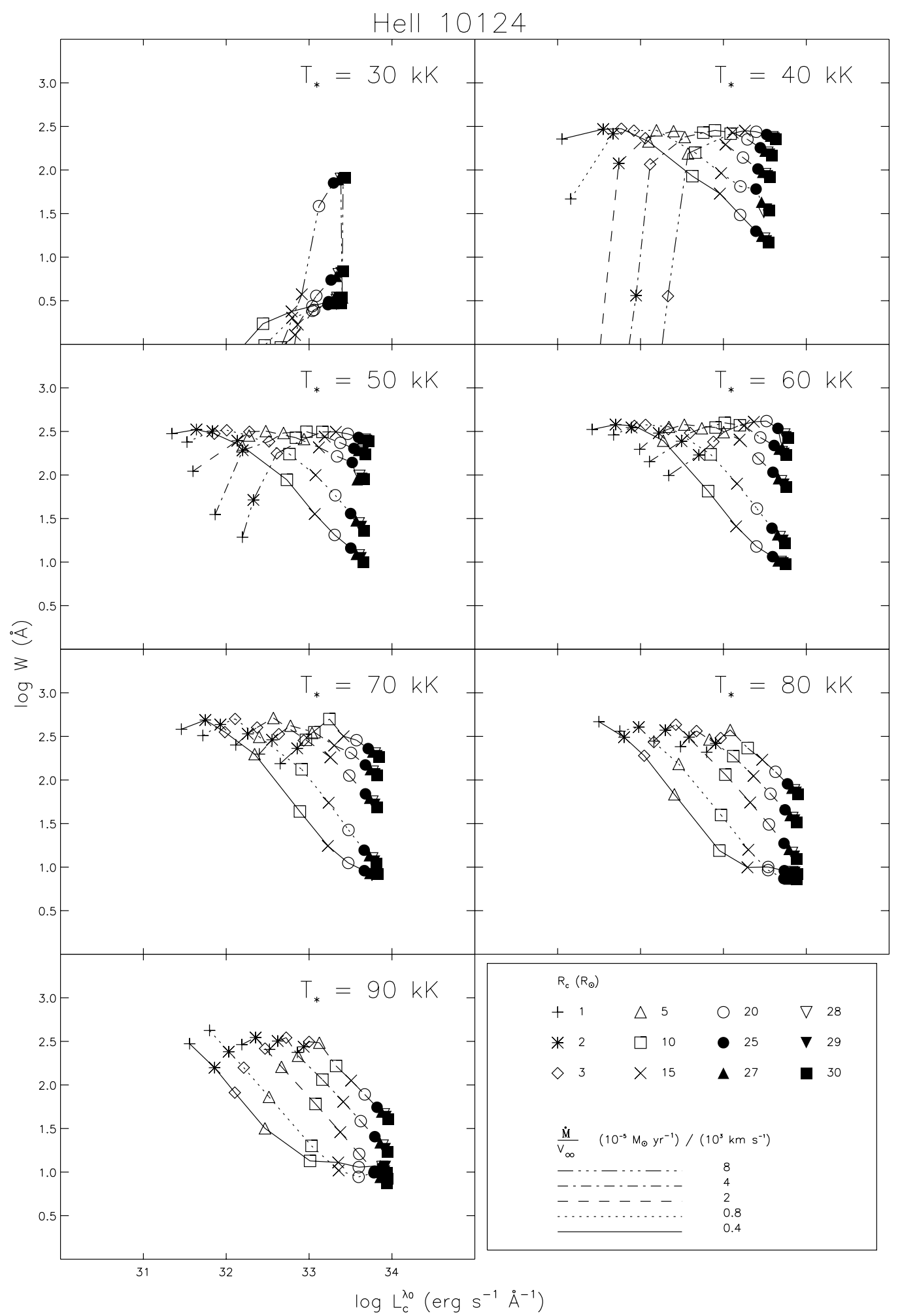

Fig. 9. Line equivalent width vs. monochromatic continuum luminosity for the He II $\lambda 10124$ line. For explanation, see the text below Fig. 5 .

effective radius to increase. Figure 5 shows that differences in mass-loss rate at constant core radius show approximately the same continuum luminosity (roughly given by Eq. (8)). This is because the increase of $x_{c}$ with mass loss rate is compensated by the lower temperature at $x_{\mathrm{c}}$. At large wavelength and for dense winds, however, $x_{\mathrm{c}}$ is large enough to lie in the isothermal regions of the wind, so the increase of $x_{\mathrm{c}}$ leads to a higher continuum luminosity. 
In Sect. 2 we saw that if the continuum is formed in the wind, the Baldwin relations are steeper at higher densities, due to the influence of the continuum effective radius on the emission measure (see Fig. 2 and the text in Sect. 2.2). This behaviour is opposite from what is seen in the detailed model calculations, where the relations appear to flatten when going to higher density (i.e. smaller core radius), as seen in Figs. 5 to 9. The reason for this lies in the line optical depth. As mentioned in paragraph 2.3, for optically thick lines, the effective radius for the line, $x_{1}$, can be (much) larger than the continuum effective radius, $x_{\mathrm{c}}$, reducing the line emission measure. Indeed, for high wind densities the lines can be optically thick. For the He II $\lambda 1640$ line, this is shown in Fig. 10, which depicts the tangential Sobolev optical depth (Eq. (18)) of the model atmospheres at a radius of $r=1.5 R_{\mathrm{c}}$. The increasing line optical depths, visible in denser winds, explains the flattening of the Baldwin relations.

We conclude from the detailed model calculations that the Baldwin effect in He II recombination lines is a general phenomenon in Wolf-Rayet stars, where the continuum radiation field is formed in the wind. For thin winds (small mass flux) the Baldwin relations breaks down, due to the steep temperature rise at the base of the wind. Also for very thick and cool winds, there is no Baldwin effect, as recombination from He III to He II weakens the line emission.

\subsection{Effective temperature, $T_{\text {eff, }}$, versus stellar temperature, $T_{*}$}

In the above description of the Baldwin effect for WolfRayet stars, we presented the results for different values of the stellar temperature, $T_{*}$ (defined in Eq. (26)). As mentioned before, this temperature does not equal the effective temperature, $T_{\text {eff }}$ (see Sect. 3.2). Furthermore, the stellar temperature is a quantity that cannot be derived from observations, whereas the effective temperature can. In applying the Baldwin relations one would therefore rather present them for certain values of $T_{\text {eff }}$ than for $T_{*}$.

To get a feeling of how a representation for different values of $T_{\text {eff }}$ (instead of $T_{*}$ ) alters the Baldwin relations, Fig. 11 shows again the relation between line equivalent width and monochromatic continuum luminosity, for the He II $\lambda 1640$ line, this time as function of $T_{\text {eff }}$ instead of $T_{*}$.

Note, however, that the value of $T_{\text {eff }}$ is not an input parameter, but result from the model output. Because the value of $T_{\text {eff }}$ essentially depends on wind density, a sample of Wolf-Rayet star models with the same value of $T_{*}$ will cover a range in $T_{\text {eff }}$. Each panel in Fig. 11 groups together those models for which the value of $T_{\text {eff }}$ is within $5000 \mathrm{~K}$ from the value indicated.

Clearly, the largest differences between Fig. 11 (as function of $T_{\text {eff }}$ ) and Fig. 5 (as function of $T_{*}$ ) are at the lowest and highest values of $T_{\text {eff }}$. Since $T_{\text {eff }}<T_{*}$, and the highest value of $T_{*}$ is $90 \mathrm{kK}$, there are only a few models with $T_{\text {eff }}=90 \mathrm{kK}$, whereas there are many

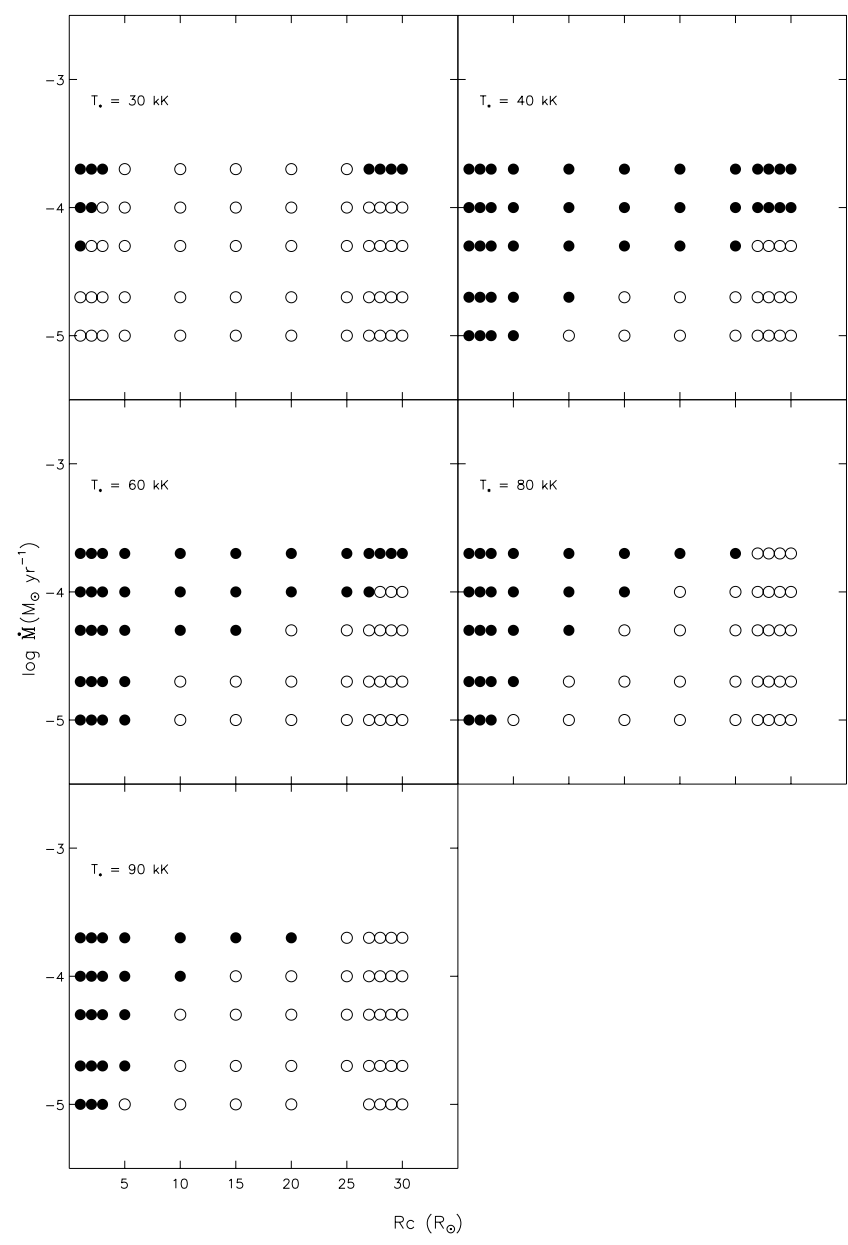

Fig. 10. Tangential Sobolev optical depth, $\tau_{\tan }$, for the He II $\lambda 1640$ line, as function of core radius ( $x$-axis) and mass-loss rate ( $y$-axis) for different stellar temperatures. Open circles represent models with $\tau_{\text {tan }}<1$, full circles represent models with $\tau_{\tan }>1$. For $T_{*}=30 \mathrm{kK}$, the line is optically thick in the top-right models. This is because the line emission is relatively strong, as a result of both the high mass-loss rate and high ionization due to the large core radius.

models with $T_{\text {eff }}$ around $30 \mathrm{kK}$. For the latter no Baldwin effect is visible, because, although for those models $T_{\text {eff }}$ lies around $30 \mathrm{kK}$, they have different stellar temperatures, $T_{*}$, and therefore show different degrees of ionization in the line forming region. Despite all this, the relation between equivalent width and continuum luminosity does not alter much when this relation is presented for a certain effective temperature instead of stellar temperature.

\subsection{Parametrizations}

To describe the Baldwin relations of our model grid, we present a set of expressions that give the continuum luminosity at the line wavelength, as a function of equivalent width and mass-loss rate, for a certain stellar temperature, $T_{*}$. Note that these equations do not provide the absolute calibration of the Baldwin effect, as our models do not yet 


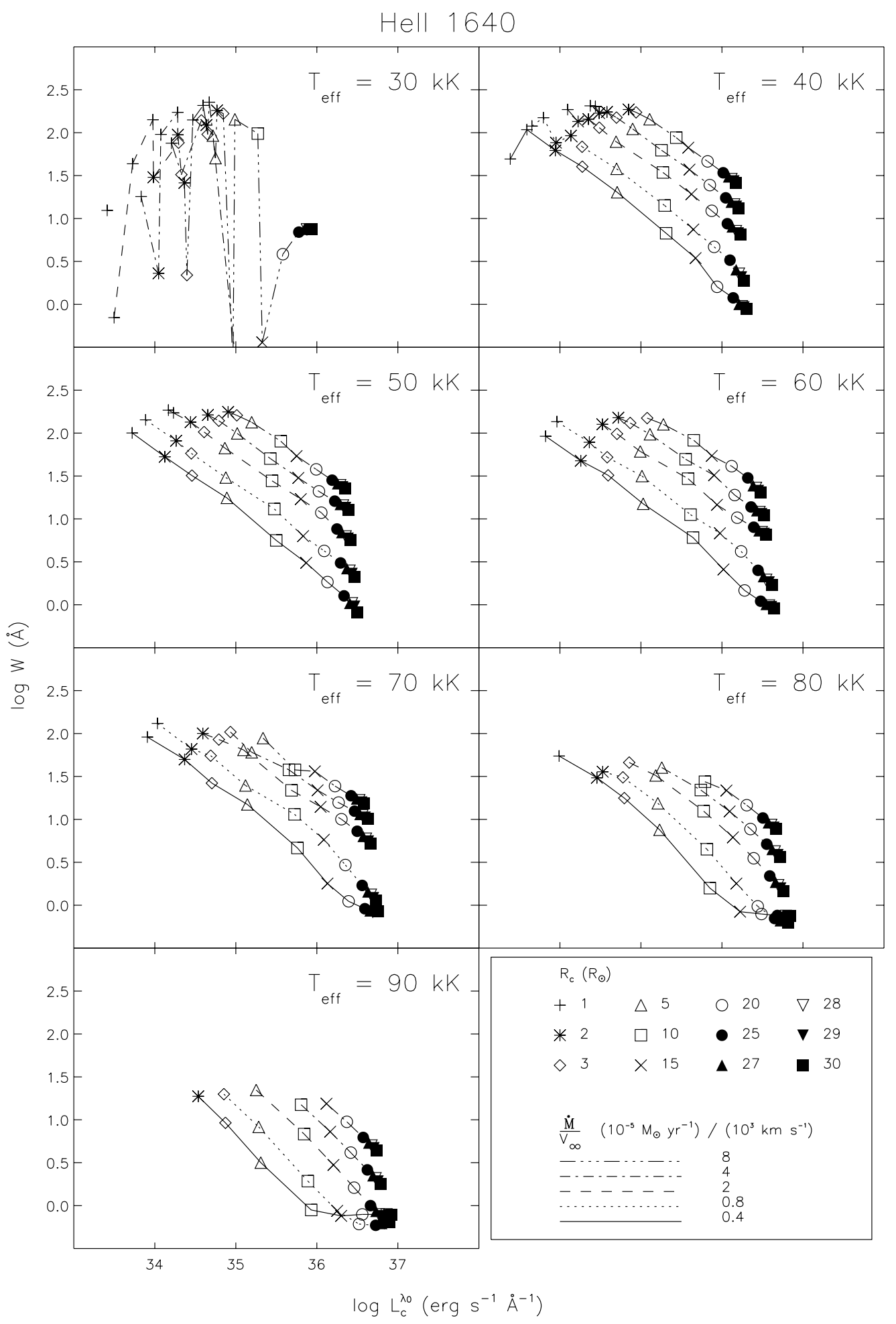

Fig. 11. The Baldwin relations of the the He II $\lambda 1640$ line, but now as a function of $T_{\text {eff }}$ rather than $T_{*}$ (compare with Fig. 5 ). Each panel shows $\log (W)$ as function of $\log \left(L_{\mathrm{c}}\right)$ for models with the same value of $T_{\text {eff }}$, and for different values of the mass-loss rate, $\dot{M}$, and the core radius, $R_{*}$. Models with the same value of $\dot{M}$ are connected by lines; the values of $R_{*}$ are indicated by symbols. Line style and symbols are explained in the bottom right panel.

contain all relevant effects such as clumping and electron scattering in line wings. As explained in Sect. 1, we prefer a somewhat simplified approach as the main aim of this paper is to investigate the basic physics of the Baldwin effect.
For each value of $T_{*}$, and each spectral line, we selected by eye the region in Figs. 5 to 9 for which $\log W$ varies more or less linearly with $\log L_{\mathrm{c}}^{\lambda_{0}}$. For these regions we applied a two dimensional linear regression technique 
Table 3. Fit coefficients and parameter range limits for the Baldwin relations as described by Eq. (28). $L_{\mathrm{c}}^{\lambda_{0}}$ is given in units of $L_{\odot} ; W$ is given in $\AA$.

\begin{tabular}{|c|c|c|c|c|c|c|c|c|}
\hline$T_{*}$ & 30000 & 35000 & 40000 & 50000 & 60000 & 70000 & 80000 & 90000 \\
\hline \multicolumn{9}{|l|}{ He II $\lambda 1640$} \\
\hline$a$ & - & -1.27 & -1.17 & -1.36 & -1.39 & -1.31 & -1.18 & -1.07 \\
\hline$\sigma_{a}$ & - & 0.07 & 0.03 & 0.02 & 0.03 & 0.04 & 0.02 & 0.03 \\
\hline$b$ & - & 1.23 & 1.20 & 1.26 & 1.25 & 1.10 & 1.16 & 1.14 \\
\hline$\sigma_{b}$ & - & 0.07 & 0.04 & 0.03 & 0.03 & 0.04 & 0.02 & 0.03 \\
\hline$c$ & - & 46.60 & 46.24 & 47.02 & 47.13 & 45.83 & 45.87 & 45.39 \\
\hline Min. $\log L_{\mathrm{c}}^{\lambda_{0}}$ & 35. & 35.2 & 35. & 34.8 & 34.9 & 35.0 & 35.1 & 34.8 \\
\hline Max. $\log L_{\mathrm{c}}^{\lambda_{0}}$ & 34. & 36.5 & 36.3 & 36.5 & 36.7 & 36.5 & 37.0 & 36.0 \\
\hline Min. $\log W$ & 0.0 & 0.5 & -0.5 & -0.5 & -0.1 & -0.1 & -0.1 & 0.0 \\
\hline Max. $\log W$ & 0.0 & 0.5 & -0.5 & -0.5 & -0.1 & -0.1 & -0.1 & 0.0 \\
\hline \multicolumn{9}{|l|}{ He II $\lambda 3203$} \\
\hline$a$ & - & -1.37 & -1.21 & -1.34 & -1.39 & -1.31 & -1.11 & -1.02 \\
\hline$\sigma_{a}$ & - & 0.09 & 0.05 & 0.05 & 0.05 & 0.03 & 0.02 & 0.02 \\
\hline$b$ & - & 1.21 & 1.17 & 1.25 & 1.29 & 1.19 & 1.09 & 1.17 \\
\hline$\sigma_{b}$ & - & 0.08 & 0.05 & 0.04 & 0.04 & 0.02 & 0.02 & 0.01 \\
\hline$c$ & - & 45.48 & 45.01 & 45.76 & 46.22 & 45.30 & 44.06 & 44.36 \\
\hline Min. $\log L_{\mathrm{c}}^{\lambda_{0}}$ & 35. & 34.3 & 34.2 & 34.5 & 34.1 & 32.9 & 32.9 & 32.9 \\
\hline Max. $\log L_{\mathrm{c}}^{\lambda_{0}}$ & 34. & 35.5 & 35.2 & 35.5 & 35.7 & 35.9 & 36.0 & 35.8 \\
\hline Min. $\log W$ & -0.5 & 0.4 & -0.1 & 0.2 & 0.2 & 0.3 & 0.4 & 0.4 \\
\hline Max. $\log W$ & 2.5 & 1.7 & 1.7 & 1.7 & 1.7 & 1.6 & 1.7 & 1.4 \\
\hline \multicolumn{9}{|l|}{ He II $\lambda 4686$} \\
\hline$a$ & - & -0.82 & -1.12 & -1.22 & -1.17 & -1.10 & -1.11 & -1.05 \\
\hline$\sigma_{a}$ & - & 0.07 & 0.06 & 0.06 & 0.06 & 0.05 & 0.02 & 0.01 \\
\hline$b$ & - & 0.87 & 1.07 & 1.19 & 1.12 & 1.07 & 1.16 & 1.20 \\
\hline$\sigma_{b}$ & - & 0.07 & 0.07 & 0.06 & 0.06 & 0.05 & 0.02 & 0.01 \\
\hline$c$ & - & 42.48 & 44.49 & 45.62 & 45.04 & 44.51 & 44.86 & 44.89 \\
\hline Min. $\log L_{\mathrm{c}}^{\lambda_{0}}$ & 35. & 34. & 34. & 34.0 & 34.0 & 34.0 & 32.3 & 32.3 \\
\hline Max. $\log L_{\mathrm{c}}^{\lambda_{0}}$ & 34. & 34.8 & 35.0 & 35.0 & 34.9 & 36.7 & 35.2 & 35.3 \\
\hline Min. $\log W$ & 2.0 & 0.4 & 0.5 & 0.5 & 0.5 & 0.5 & 0.6 & 0.6 \\
\hline Max. $\log W$ & -0.5 & 2.3 & 2.4 & 2.3 & 2.3 & 2.4 & 2.2 & 2.4 \\
\hline \multicolumn{9}{|l|}{ He II $\lambda 5411$} \\
\hline$a$ & - & -0.74 & -1.15 & -1.39 & - & - & -0.97 & -0.95 \\
\hline$\sigma_{a}$ & - & 0.04 & 0.06 & 0.07 & - & - & 0.02 & 0.03 \\
\hline$b$ & - & 1.32 & 1.01 & 1.23 & - & - & 1.08 & 1.15 \\
\hline$\sigma_{b}$ & - & 0.05 & 0.05 & 0.06 & - & - & 0.01 & 0.01 \\
\hline$c$ & - & 44.95 & 42.88 & 44.91 & - & - & 42.95 & 43.32 \\
\hline Min. $\log L_{\mathrm{c}}^{\lambda_{0}}$ & 35. & 33.4 & 33.9 & 33.6 & 34.4 & 34.0 & 32.1 & 32.1 \\
\hline Max. $\log L_{\mathrm{c}}^{\lambda_{0}}$ & 34. & 34.3 & 34.8 & 34.8 & 34.0 & 34.0 & 35.0 & 35.0 \\
\hline Min. $\log W$ & 2.0 & -0.2 & 0.1 & 0.2 & 0.3 & 0.3 & 0.5 & 0.7 \\
\hline Max. $\log W$ & -1.5 & 1.0 & 1.5 & 1.5 & 1.5 & 1.5 & 1.4 & 1.4 \\
\hline \multicolumn{9}{|l|}{ He II $\lambda 10124$} \\
\hline$a$ & - & -0.96 & -1.13 & - & -0.97 & -0.86 & -0.96 & -1.00 \\
\hline$\sigma_{a}$ & - & 0.03 & 0.05 & - & 0.03 & 0.02 & 0.02 & 0.02 \\
\hline$b$ & - & 1.39 & 1.17 & - & 1.39 & 1.27 & 1.16 & 1.21 \\
\hline$\sigma_{b}$ & - & 0.05 & 0.05 & - & 0.04 & 0.02 & 0.01 & 0.01 \\
\hline$c$ & - & 46.00 & 44.67 & - & 46.19 & 45.02 & 43.85 & 44.10 \\
\hline Min. $\log L_{\mathrm{c}}^{\lambda_{0}}$ & 35. & 32.6 & 32.6 & 32.6 & 32.6 & 32.7 & 32.2 & 32.0 \\
\hline Max. $\log L_{\mathrm{c}}^{\lambda_{0}}$ & 34. & 33.6 & 33.8 & 32.0 & 33.9 & 33.9 & 34.0 & 34.0 \\
\hline Min. $\log W$ & 3.5 & 0.7 & 1.0 & 1.0 & 1.0 & 1.0 & 1.1 & 1.3 \\
\hline Max. $\log W$ & 0.0 & 2.0 & 2.0 & 2.0 & 2.1 & 2.1 & 2.3 & 2.2 \\
\hline
\end{tabular}


to express $\log L_{\mathrm{c}}^{\lambda_{0}}$ as function of $\log W$ and $\log \left(\dot{M} / v_{\infty}\right)$. The result is a set of equations of the form

$\log L_{\mathrm{c}}^{\lambda_{0}}=a\left(T_{*}\right) \log W+b\left(T_{*}\right) \log \frac{\dot{M}}{v_{\infty}}+c\left(T_{*}\right)$,

with $W$ in $\AA, \dot{M}$ in $M_{\odot} \mathrm{yr}^{-1}$, and $v_{\infty}$ in $\mathrm{km} \mathrm{s}^{-1}$.

The limits of the range where Eq. (28) applies, as well as the temperature dependent fit coefficients $a\left(T_{*}\right)$ and $b\left(T_{*}\right)$ are given in Table 3.

The best results are obtained when applying the expressions for as many spectral lines as possible, taking into account the quality of the line features in the considered spectrum. For some stellar temperatures no data can be given, since for some equivalent width values more than one solution is possible for the continuum luminosity. In other words: for those stellar temperatures, the area in the $\log W-\log L_{\mathrm{c}}^{\lambda_{0}}$ plane where the Baldwin relations behave linearly, is too small to provide useful parametrisations.

\section{Discussion}

Extensive studies exist on the behaviour of the equivalent width of spectral lines and continuum luminosity in WolfRayet spectra with basic stellar parameters (e.g. Hamann \& Koesterke 1998; Nugis et al. 1998). Morris et al. (1993). were the first to show a relation between the two quantities for Wolf-Rayet stars. The purpose of our study is mainly to indicate the existence of the Baldwin effect for almost the whole range of Wolf-Rayet stellar parameters and to explain the physics that cause the effect. The model calculations have shown that the effect is mainly caused by differences in radius among the stars. Differences in stellar temperature and mass-loss rate cause a broadening of the relation. Variations of the normalized effective radius $x_{\mathrm{c}}$ cannot cause the Baldwin effect (as was proposed by Morris et al. 1993) but do determine the slope of the Baldwin relations. The ionization structure determines the parameter region where the Baldwin effect applies.

The fact that sufficiently dense atmospheres are required in order to show a Baldwin effect, explains why the effect has been observed in Wolf-Rayet stars and not in normal O-stars. Indeed, for O-stars the continuum spectrum is not formed in the wind, except for wavelengths in the infrared and longer. We calculated also a few Ostar models, i.e., with parameters similar to our WolfRayet star model grid, but with lower mass-loss rates: $-7<\log (\dot{M})<-5.5$. We found no clear correlation between line equivalent width and monochromatic continuum luminosity.

The influence of stellar temperature, and mass-loss rate, has implications for the applicability of the Baldwin effect in the determination of stellar distances. The broadening of the $W-L_{\mathrm{c}}$ relation is mainly caused by differences in the mass-loss rate. This indicates that a star's absolute luminosity (and through that it's distance) is difficult to obtain from a single relation between equivalent width and continuum luminosity due to this intrinsic spread. Indeed, without some knowledge of a star's mass-loss rate (which already depends on it's luminosity!), we cannot guarantee to derive a distance from a single equivalent width versus $L_{\mathrm{c}}$ relation with error bars systematically any smaller than currently found in the literature. However, it may be possible to define separate equivalent width versus $L_{\mathrm{c}}$ relations for the three groups of late-type, strong-lined earlytype, and weak-lined early-type WN stars. These groups tend to cluster in parameter space (see, e.g., Hamann \& Koesterke 1998), and will thus occupy limited regions of our grids of Baldwin relations for the He II lines.

Before making use of the Baldwin relations, however, we must fully account for deficiencies in our model calculations that will contribute to uncertainties in the equivalent width measurements. The line equivalent width in the stellar spectra may be influenced by additional Doppler broadening arising from unaccounted for turbulent motion in, e.g., shocks. Also, the neglect of nitrogen has a slight influence on the He line profiles (Hamann \& Koesterke 1998). Over recent years, furthermore, it has become more obvious that the spherically symmetric, homogeneous approach in model atmosphere calculations does not suffice to describe spectral observations; wind clumping phenomena may influence both the line profile shape (in the electron scattering wings) and the overall SED, as shown by Hillier (1984, 1991). Observational evidence for clumping can be found in e.g. Lépine \& Moffat (1999). The impact of clumping in terms of the stellar parameters is chiefly in the estimated values of $\dot{M}$, and may therefore alter the manner in which the Baldwin relations are applied on a source by source basis.

In a forthcoming paper we will treat some of these effects, such as the inclusion of nitrogen and influence of clumping. The final calibration of the Baldwin relations will be done by means of observations of stars with well known distances. This will set the stage for application of the Baldwin effect to Galactic WR star observations.

Acknowledgements. AdK acknowledges support from NWO Pionier grant 600-78-333 to L. B. F. M. Waters and from NWO Spinoza grant 08-0 to E. P. J. van den Heuvel. We thank Dr. Richard Ignace for his useful comments and suggestions.

\section{References}

Baldwin, J. A. 1977, ApJ, 214, 679

Brussaard, P. J., \& van de Hulst, H. C. 1962, Rev. Mod. Phys., 34,507

Conti, P. S., \& Underhill, A. B. 1988, O stars and Wolf-Rayet stars, Monograph Series on Nonthermal Phenomena in Stellar Atmosphere - NASA SP, Washington: NASA, 1988, ed. P. S. Conti, \& A. B. Underhill

de Koter, A., Schmutz, W., \& Lamers, H. J. G. L. M. 1993, A\&A, 277, 561

de Koter, A., Heap, S. R., \& Hubeny, I. 1997, ApJ, 477, 792

Drew, J. E. 1989, ApJS, 71, 267 
Hamann, W.-R. 1985, A\&A, 145, 443

Hamann, W.-R., \& Koesterke, L. 1998, A\&A, 333, 251

Hamann, W.-R., Wessolowski, U., \& Koesterke, L. 1994, A\&A, 281,184

Hillier, D. J. 1984, ApJ, 280, 744

Hillier, D. J. 1991, A\&A, 247, 455

Lamers, H. J. G. L. M., \& Waters, L. B. F. M. 1984, A\&A, 136,37

Lamers, H. J. G. L. M., \& Cassinelli, J. P. 1999, Introduction to stellar winds (Cambridge University Press)

Leitherer, C. 1988, ApJ, 326, 356

Lépine, S., \& Moffat, A. F. J. 1999, ApJ, 514, 909

Morris, P., Conti, P. S., Lamers, H. J. G. L. M., \& Koenigsberger, G. 1993, ApJ, 414, L25
Morris, P. W. 1995, Ph.D. Thesis, University of Colorado at Boulder

Nugis, T., Crowther, P. A., \& Willis, A. J. 1998, A\&A, 333, 956

Puls, J., \& Hummer, D. G. 1988, A\&A, 191, 87

Schmutz, W., Hamann, W.-R., \& Wessolowski, U. 1989, A\&A, 210,236

Schmutz, W., Abbott, D. C., Russell, R. S., Hamann, W. R., \& Wessolowski, U. 1990, ApJ, 355, 255 van der Hucht, K. A. 2001, NewAR, 45, 135

van der Hucht, K. A., Hidayat, B., Admiranto, A. G., Supelli, K. R., \& Doom, C. 1988, A\&A, 199, 217

Waters, L. B. F. M., \& Lamers, H. J. G. L. M. 1984, A\&AS, 57, 327 